\section{MedienPädagogik}

www.medienpaed.com
Zeitschrift für

Theorie und Praxis

der Medienbildung

ISSN 1424-3636

Themenheft Nr. 15/16: Computerspiele und Videogames

in formellen und informellen Bildungskontexten

\title{
Bildungspotenziale digitaler Spiele und Spielkulturen
}

Johannes Fromme, Benjamin Jörissen, Alexander Unger

\begin{abstract}
Der Beitrag konzentriert sich auf den Bereich des informellen und selbstgesteuerten Lernens im Kontext von Computerspielen und Computerspielkulturen und verfolgt das Ziel, ausgehend von einer Untersuchung ausgewählter Bildungspotenziale Grundlagen für die Einschätzung und Bewertung von Computerspielen sowie ihren Einsatz in pädagogischen Kontexten anzubieten. Es wird dazu zunächst begrifflich zwischen Lernen und Bildung unterschieden, um einer engen instrumentellen Perspektive zu entkommen. Anschliessend werden unterschiedliche Arten von digitalen Bildungsräumen thematisiert, bevor spezifische Bildungspotenziale von Singleplayer-Spielen und sowie von community-basierten Multiplayer-Spielen genauer erläutert und diskutiert werden. Abschliessend plädiert der Beitrag dafür, die Kluft zwischen formalen und informellen Lernumgebungen zu überbrücken.
\end{abstract}

\section{Einleitung}

In öffentlichen Diskussionen werden Computer- und Videospiele nach wie vor zumeist misstrauisch beurteilt, weil ihnen negative Effekte wie eine Steigerung der Aggressivität, Förderung sozialer Isolation oder suchtartige Verhaltensweisen unterstellt werden. Die öffentliche Debatte hält damit allerdings nicht Schritt mit neueren Entwicklungen und Erkenntnissen im wissenschaftlichen Kontext, die differenziertere Einschätzungen nahelegen. Seit den späten 1990er Jahren ist eine Zunahme an empirischen Studien und theoretischen Arbeiten zu Computerspielen zu verzeichnen (vgl. Greenfield \& Cocking 1996; Fritz \& Fehr 1997; Fromme, Meder \& Vollmer 2000; Raessens \& Goldstein 2005; Vorderer \& Bryant 2006). Zu konstatieren ist damit eine beginnende Etablierung einer interdisziplinär angelegten und über eine enge Wirkungsperspektive hinausweisenden Computerspielforschung (vgl. Fromme 1997). Vor diesem Hintergrund scheint es auch eine «renewed awareness of the potential of simulations and games among researchers interested in learning and cognition» zu geben (Arnseth 2006), welche sich vor allem auf Diskussionen und Projekte im Bereich des sog. "Serious Gamings», also des «ernsthaften» Einsatzes von Computerspielen in Kontexten der Erziehung, Weiterbildung, Gesundheit usw. bezieht.

Diese Diskussion möchten wir aufgreifen, dabei aber eine etwas anders gelagerte Perspektive präsentieren: Computerspiele beinhalten unseres Erachtens häufig Bildungspotenziale, die in den gegenwärtigen Diskussionen um Serious Gaming und Lernspiele zumeist übersehen werden. «Bildung» ist in diesem Fall nicht im 
alltagssprachlichen Sinne von Ausbildung oder Lernen angesprochen, sondern im Sinne der humanistischen Bildungstheorie als selbstreflexiver Prozess der Veränderung von Welt- und Selbstreferenzen. Es geht mithin um Prozesse der Flexibilisierung von Weltsichten und des Aufbaus von Orientierungswissen in komplexer werdenden gesellschaftlichen Welten und Situationen. Die sozialen Strukturen und Praxen des Computerspielens besser kennenzulernen bedeutet daher aus unserer Sicht, wichtige Einsichten in die Grenzen und Möglichkeiten des Einsatzes von Computerspielen für pädagogische Ziele und Zwecke zu erhalten, die über eine blosse Instruktions- und Trainingsperspektive hinausweisen. Damit geht zugleich die Einsicht einher, dass in diesem Feld Prozesse des informellen, selbstgesteuerten Lernens dominieren, und dass eine lohnenswerte Forschungsaufgabe aus medienpädagogischer Sicht darin besteht, die sozialen Dynamiken dieser Prozesse genauer zu erfassen.

Der Beitrag erläutert zunächst das bereits angesprochene Verständnis von Bildung und von Lernen und thematisiert in einem zweiten Abschnitt unterschiedliche Arten von Bildungsräumen, die sich in digitalen Spielen finden lassen. Die Bildungspotenziale von Computerspielen werden anschliessend zunächst an SingleplayerSpielen und danach an community-basierten Multiplayer-Spielen erläutert. Der letzte Abschnitt fasst die wichtigsten Aspekte der Diskussion zusammen und versucht, einige Grundlagen zur Einschätzung des Bildungspotenzials von Computerspielen in bildungsbezogenen Kontexten anzubieten, wobei letztlich dahingehend argumentiert wird, die Kluft zwischen formalen und informellen Lernumgebungen zu überbrücken.

\section{Lernen vs. Bildung}

Da heute fast alle jungen (und zunehmend mehr ältere) Menschen in Kontakt mit Computerspielen stehen, ist es nicht verwunderlich, dass verstärkt auch ein instrumentelles Interesse an diesem Phänomen entsteht, das in der Frage nach der Möglichkeit mündet, Computerspiele(n) für pädagogische und andere Zwecke zu nutzen. Dies ist durchaus nachvollziehbar und legitim. Allerdings besteht dabei eine Tendenz, den Zusammenhang von Computerspielen und Lernen/Bildung sehr eng zu fassen und vor allem informelle Prozesse zu vernachlässigen. Unser Plädoyer geht dahin, die enge instrumentelle Perspektive auf Computerspiele erheblich zu erweitern und ein besseres Verständnis informeller Lern- und Bildungsprozesse in digitalen Computerspiel(er)-Kulturen anzustreben.

Dabei wird einerseits die aktive und konstruktive Rolle des Individuums in Lernund Bildungsprozessen stärker in den Blick genommen. Bereits die klassische Bildungstheorie betonte bekanntermassen, dass Bildungs- und Subjektivierungsprozesse auf der permanenten Auseinandersetzung des Einzelnen mit verschiedenen kulturellen Welten basieren. Wenn man diese aktive Rolle ernst nimmt und anerkennt, muss man zugleich zugestehen, dass formelle und institutionalisierte 
Lernsettings - wie etwa die Schule - nur einen speziellen Typ einer Lernumgebung darstellen.

Andererseits wird damit eine Auffassung von Bildung relevant, die sowohl über Ausbildung als auch über "Gebildetheit» im Sinne eines Bildungskanons weit hinausgeht. Bildungsprozesse sind solche, in denen das Individuum in der kulturellen und sozialen Partizipation Erfahrungen macht, die (im günstigen Fall) zur Reflexivierung und Dezentrierung, somit zur Flexibilisierung und Bereicherung seiner hergebrachten Muster der Selbst- und Weltaufordnung beitragen (Marotzki 1990). Dabei spielen kreative und spielerische Freiräume der Artikulation, wie etwa in Spielen oder in ästhetischen Tätigkeiten, eine ebenso grosse Rolle wie die Konfrontation mit der eigenen und mit fremden Sprachen, Kulturen und Gewohnheiten.

Was wir also hier unter Bildung verstehen wollen, sind selbstinitiierte Prozesse, die den Horizont der alltäglichen Weltsicht überschreiten und die Art und Weise, wie Individuen ihrer Welt «Sinn geben» und wie sie sich selbst verstehen (Identität), verändern. Bildungsprozesse in diesem Sinne können nicht «von aussen», also etwa durch Lehrer oder Erzieher, herbeigeführt werden. Allerdings weist jeder Erfahrungsraum Anreize und Potenziale auf, die zu einer solchen Reorganisation von Welt- und Selbstsichten führen können. Dies gilt zumal für mediale Artikulationsräume (vgl. Jörissen/Marotzki 2008). Im Hinblick auf die besondere Bedeutung von Selbstreflexivität und Orientierung ist es evident, dass jede Gelegenheit, eine tentative Haltung zur Welt zu erlangen, von hohem pädagogischen Wert ist. Obwohl es nicht möglich ist, Bildungsprozesse in diesem anspruchsvollen Sinn unmittelbar zu forcieren, können pädagogische Handlungs- und Gestaltungsformen durchaus dazu beitragen, Bildungsprozesse zu ermöglichen, zu erleichtern und zu provozieren, etwa indem entsprechende Umgebungen und Erfahrungsräume anregend gestaltet werden. Im Folgenden möchten wir aufzeigen, dass und inwiefern digitale Spiele und Spiel(er)-Kulturen als neuer Teil der Alltagswelt von Kindern, Teenagern und Erwachsenen als Umgebungen für Bildungsprozesse betrachtet werden können.

\section{Computerspiele als Kultur- und Bildungsräume}

In der sozialwissenschaftlichen Debatte wird heute kaum mehr bestritten, dass Technik zunehmend unsere Lebenswelt durchdringt und mehr oder weniger zu einem Hintergrund (post-) moderner Gesellschaften geworden ist. In welcher Weise Technik und besonders die Neuen Medien das soziale und kulturelle Leben beeinflussen, wird gegenwärtig kontrovers diskutiert. Dabei wird ein entscheidender Unterscheid zwischen so genannten ‘alten` und «neuen` Medien immer wieder hervorgehoben: Traditionelle (Massen-)Medien basieren auf einem undirektionalen Kommunikationsmodell, bei dem der Nutzer Teil eines Publikums ist und Botschaften empfängt (Sender-Empfänger-Modell). Zwar kann auch das Emp- 
fangen und dekodieren von Botschaften als ein aktiver Prozess verstanden werden (vgl. Hall 1980), da die Interpretation einer Nachricht nur teilweise durch den Empfänger determiniert werden kann. Letztlich bleibt der Nutzer aber in der Rolle des Rezipienten (vgl. Rösler 2004; Jäckel 2008). Aus Sicht der Rezipienten haben die ‘alten` Medien den Zugang zu Wissen und damit auch zu unserer Welt entscheidend erweitert und so vielfältige soziokulturelle Umbrüche forciert. Massenmedien ermöglichen es u. a., sich über Weltbereiche und Kulturen zu informieren, die weit ausserhalb der «natürlichen` Reichweite eines Individuums liegen, so dass der Einzelne über Medien ein nicht unerhebliches Wissen über diese erweiterte Umwelt gewinnen kann. Die Möglichkeiten mit diesen Weltbereichen zu interagieren und 〈reale) Eindrücke von ihnen zu erhalten, sind mit traditionellen Massenmedien allerdings begrenzt.

Genau in dieser Hinsicht schaffen die «neuen` Medien neue Möglichkeiten. Ihre simulativen Potenziale erlauben es den Nutzern näher an das heranzukommen, was man als «reale) Erfahrung bezeichnen würde. Durch Vernetzung, Interaktivität und Simulation können die Neuen Medien virtuelle Interaktionsräume schaffen, in denen sich neue Formen der Interaktion und Kommunikation ausbilden und realitätsnahe Erfahrungen mit unterschiedlichen (digitalen) Gegenständen und Umgebungen gewonnen werden können (vgl. Fromme 2008). Die neuen Medien haben in diesem Sinne interaktive Welten erschaffen - Welten, die oft durch Tentativität und Ludizität gekennzeichnet sind und eigene Subkulturen hervorbringen können. Dazu gehören z. B. auch die Welten, die uns Offline- und Online-Computerspiele zur Interaktion und zur Immersion anbieten. Diese neuen, interaktiven Erfahrungsräume sind aber nicht als ein gegenüber der «realen` Welt abgeschlossener Bereich zu verstehen, wie es die Dichotomie von real und virtuell bzw. von Simulation und Realität nahe legt. Vielmehr zeigt sich, dass sich mit der technischen Durchdringung des sozialen und kulturellen Lebens (vgl. Jenkins 2006; Poster 2006) auch virtuelle und reale (Erfahrungs-)Räume zunehmend vermischen. Ganz konkret sichtbar wird das etwa, wenn sich Clans aus Online-Rollenspielen im «Real-Life» treffen. «Kultur» und "Sozialität» dehnen sich in diesem Sinne zunehmend auch in die interaktiven Medienwelten aus (vgl. Fromme 2006). Die medialen Welten, ihre Praktiken und Rituale, werden zunehmend zu einem integralen Bestandteil der alltäglichen Lebenswelt.

Die Neuen Medien können vor diesem Hintergrund als ein Transformator bzw. als ein transformativer Raum verstanden werden, in dem traditionelle soziale Praktiken erneuert und restrukturiert werden sowie neue Sozialformen entstehen (vgl. Gamm 2000; Jones 1997). Dieser Prozess zeigt sich allerdings als durchaus ambivalent: die Transformation und das «Framing» sozialer Praxis kann einerseits zu einer Öffnung und Erweiterung von Handlungsoptionen führen, andererseits aber auch zu Restriktion oder zur Tilgung von Freiheitsgraden, z. B. durch ein Übermass an Steuerung - ein Problem, das wir u. a. aus dem Bereich des E-learnings kennen 
(vgl. Unger 2007, S. 215f.). Eine (pädagogische) Einschätzung oder gar Bewertung virtueller resp. softwarebasierter Räume erfordert demnach eine differenzierte Analyse der jeweiligen virtuellen Umgebung und ihrer «Kulturen», um ihre Bedeutung für Sozialisation und Identitätsbildungsprozesse (vgl. Turkle 1999) ebenso wie für neue Formen des (Selbst-)Lernens, einschätzen zu können.

Software-basierte virtuelle Räume können dabei, je nachdem ob sie vernetzt sind oder nicht, zwei unterschiedliche Formen medial gerahmter Interaktion anbieten: zum einen die solipsistische Interaktion mit der Software und zum anderen die medial vermittelte Kommunikation zwischen zwei oder mehreren Personen (vgl. McMillan 2002a, 2002b). In diesem Kontext kommt dem Begriff «virtuell» eine doppelte Bedeutung zu: Erstens verweist er auf die Tatsache, dass die Interaktion an eine softwarebasierte Umgebung gebunden ist und folglich im Modus «leiblicher Abwesenheit» stattfindet. Zweitens impliziert er bezüglich der user-software-Interaktion, dass das soziale Gegenüber durch eine Software simuliert werden kann.

Die Bedeutung der Softwareumgebung für Interaktionsprozesse wurde auch schon in den Diskussionen um E-learning behandelt. So schlug Schulmeister (1997) vor, generell drei (pädagogische) Ebenen oder Räume einer Software zu unterscheiden:

1. Wenn wir mit Software interagieren, kommen wir zunächst mit dem «Präsentationsraum» in Berührung, also dem User-Interface, das u. a. interaktive Icons und verschiedene Inhalte anbietet (auf die Differenz zwischen Interface und Inhalt kommen wir später noch zurück) und auf dem die Interaktion grafisch repräsentiert bzw. visualisiert wird. Werden Computerspiele in der öffentlichen Diskussion kritisiert, wird zumeist auf diesen Raum und die hier dargestellten, mitunter gewalttätigen Inhalte rekurriert, während die folgenden beiden Räume, die entscheidend für die Komplexität und Potenzialität von virtuellen (Spiele-)Welten sind, ignoriert werden.

2. Auf einer tieferen Ebene liegt der «Ereignisraum». Er existiert ausschliesslich für die Zeit, in der ein User mit einer Software interagiert. Obwohl dieser Raum entsprechend «flüchtig» ist, repräsentiert der Handlungsraum die zeitliche Phase, in der User und Software verschmelzen und die Software so zu einen sozio-technischen Artefakt wird. Mit der Verbreitung der Netzwerktechnologie erhält dieser Raum zusätzlich eine soziale Dimension, da ein User mit anderen Usern in einer softwarebasierten Umgebung interagieren kann. In einer noch weiteren Perspektive können auch virtuelle Gemeinschaften, die um bestimmte Anwendungen und Spiele herum entstehen, als Teil des Ereignisraumes betrachtet werden.

3. Wie bereits erwähnt wurde, hängt die Wirkung medialer Räume einerseits von den Fähigkeiten des Nutzers ab die hier gemachten Erfahrungen zu verarbeiten, und andererseits von dem «Interaktions-Rahmen» der Software, der verschiedene Interaktionsformen ermöglichen oder verhindern kann. In diesem 
Sinne weist eine Software einen dritten Raum auf, den «Bedeutungsraum». Dieser Raum bezeichnet die Ebene der Software, auf der der Programmcode eingeschrieben ist, der dem Darstellungsraum zu Grunde liegt und diesen «rahmt». Hier liegen, metaphorisch gesprochen, die «Wurzeln» der «Blüten», die auf der Oberfläche des Darstellungsraums erscheinen (vgl. Schulmeister 2007). Zu dieser Ebene gehören neben dem Softwarecode auch die gespeicherten Inhalte, relationale Verknüpfungen sowie die Interaktions-Skripte. Er korrespondiert insofern mit dem, was in der Computerspielforschung als Regelsystem angesprochen wird (vgl. etwa Juul 2005).

Obwohl dieses Konzept zur Analyse von E-learning-Software entwickelt wurde, kann es (aufgrund seines hohen Abstraktionsgrades) ebenso auf den Bereich der Computerspiele und ihre virtuellen Umgebungen angewandt werden. Basierend auf diesem Modell können wir die These aufstellen, dass die Frage, ob eine Software ein «facilitating environment» (Winnicott 1990) für Lern- Bildungsprozesse darstellt, von den folgenden vier Aspekten abhängt:

- Dem Code-Raum und den dort implementierten Skripts,

- der Offenheit dieses Raumes,

- dem dargebotenen Content und seinem Lern- oder auch Irritationspotenzial, und schliesslich

- «transzendierenden» Formen der user-to-user-Interaktion, wie wir sie von Gaming oder Modding-Communities kennen (Unger 2007).

Wie diese Analyse zeigt, impliziert die Aneignung von virtuellen Umgebungen einen komplexen Prozess der Navigation, Interaktion, Selektion und Interpretation, der eine simple Sender-Empfänger-Struktur bei weitem überschreitet.

Aus bildungswissenschaftlicher Sicht können restriktiv gestaltete Umgebungen für die individuelle Entwicklung ebenso nachteilig sein wie zu komplexe Räume, welche die Lernenden überfordern. Zugleich fördert der Umgang mit Komplexität (im Sinne von Multiperspektivität und «polyphonen» Rationalitäten) wesentlich diejenigen komplexen Formen des Lernens, die wir als Bildungsprozesse bezeichnen. Grundsätzlich halten virtuelle Umgebungen ein reichhaltiges Potenzial in Bezug auf Bildungsprozesse bereit. Ob dieses letztlich freigesetzt werden kann oder nicht, hängt von ihrem Design, den implementierten Strukturen und Interaktionsoptionen sowie auch von den Prädispositionen der Nutzer ab. Vor diesem Hintergrund erweist sich ein pauschales Urteil über den Bildungswert (oder auch die Wertlosigkeit) von virtuellen Umgebungen, wie wir sie bei Computerspielen vorfinden, als verfehlt. Vielmehr zeigt es sich als Notwendigkeit, die strukturellen Aspekte im Sinne eines «close readings» in jedem einzelnen Fall zu analysieren und nach wiederkehrenden Mustern wie Besonderheiten zu suchen. 


\section{Singleplayer-Spiele als «strukturelle Generatoren» von informellen Lern- und Bildungsprozessen}

Wir können nun einen genaueren Blick auf spezifische Bildungspotenziale von Singleplayer-Spielen als eine bestimmte Form virtueller Umgebungen werfen. Wie wir aufgezeigt haben, verstehen wir unter Bildung komplexe, selbstreflexive informelle Lernprozesse. Der Begriff des informellen Lernens umfasst sowohl intendierte als auch nicht intendierte und sogar unbewusste Formen des nicht formalisierten Lernens. Die Bedeutung des informellen Lernens für Bildung und Erziehung ist lange Zeit unterschätzt worden (obwohl sie bereits 1972 in Edgar Faures UNESCO-Report deutlich aufgezeigt wurde), wird seit einigen Jahren aber breiter anerkannt und diskutiert, vor allem im Bereich der beruflichen Weiterbildung (Coffield 2000; Foley 1999; Harrison 2003; Overwien 1999).

Auf welche Weise und mit welchen Mitteln tragen Computerspiele nun zum informellen Lernen bei, und inwiefern kann man sagen, dass sie die Grenze des spielimmanenten Lernens in Richtung auf Bildungsprozesse überschreiten? In der kurzen Geschichte der Digital Game Studies lassen sich hierzu zwei prominente Positionen unterscheiden. Der erste Ansatz versteht Computerspiele als Agenten von Anpassungs- und Sozialisationsprozessen, der zweite betrachtet sie als herausfordernde Umgebungen, welche die Herausbildung bestimmter, vor allem kognitiver Fähigkeiten provozieren. Beide Ansätze gehen von spezifischen Strukturmerkmalen der digitalen Spiele aus, die in der einen oder anderen Weise ‘wirksam» werden. Anschliessend wird eine dritte Position vorgeschlagen, die Computerspiele als Lernumgebungen betrachtet, in denen auch kreative und reflexive Prozesse der Aneignung und Transformation von Regeln angeregt werden und stattfinden.

\section{Computerspiele als Sozialisationsinstanz}

Der erste Ansatz führt zunächst zu einer eher kritischen Einschätzung der Bildungspotenziale von Computerspielen. Ein frühes Beispiel dafür liefert Behn (1984). Seine Überlegungen basieren auf der Annahme, dass beim Videospiel das Spiel durch den maschinenhaften Charakter des Computers überformt wird. Die Anforderungen der Computerspiele lösen demnach zwar Lernprozesse auf Seiten des Spielers aus, diese werden aber negativ bewertet, da es sich lediglich um eine Anpassung an die Logik des Computers handele. Insofern konstituierten Computerspiele einen Lernprozess, bei dem instinktive und unmittelbare Handlungsreaktionen erforderlich seien, gleichzeitig aber emotionale Reaktionen und jedes Nachdenken unterdrückt werden müssten. Die negative Bewertung der unterstellten Sozialisationsfunktion der Computerspiele ergibt sich für Behn auch aus einer gesellschaftskritischen Grundhaltung: Die Gesellschaft trägt für ihn aufgrund zunehmender Leistungsorientierung, einer Mediatisierung der Erfahrung und der Maschinisierung vielfältigster Prozesse inhumane Züge, und Computerspiele tra- 
gen für ihn zur unbewussten Anpassung an diese Verhältnisse statt zur Emanzipation bei.

Auch Rogge (1985) diskutiert die sozialisatorische Bedeutung von Computerspielen, jedoch auf der Basis einer komplexeren theoretischen Rahmung, nämlich des Symbolischen Interaktionismus. Er betont die Relevanz der subjektiven Interpretationen und Bedeutungszuweisungen der Nutzer, die damit ihre individuelle (Medien-) Realität erschaffen. Auf der Basis von Interviews mit jungen Computerspielern zeigt Rogge auf, dass digitale Spiele eine wichtige Rolle für die Entwicklung unabhängiger Jugendkulturen spielen, u. a. weil sie es ermöglichen, sich von der Symbolwelt der Erwachsenen demonstrativ abzugrenzen. Während diese Forschungsergebnisse aufzeigen, dass Computerspiele durchaus kreative Potenziale enthalten, bezweifelt Rogge, ob sie auch die Mittel bereitstellen, eine hinreichende Distanz (zur «Mikroelektronik» und zum digital erzeugten Spielgeschehen) zu entwickeln, wie sie für metakognitive und reflexive Prozesse nötig ist. Allerdings sieht Rogge auch, dass dieser Zweifel aus einer Erwachsenenperspektive heraus erfolgt, während der unvoreingenommene Blick auf die medialen Praxen der Jugendlichen Indizien dafür liefere, dass diese keineswegs technologisch determiniert seien, sondern durchaus eigensinnige und kreative Momente aufwiesen.

\section{Kompetenzentwicklung durch Computerspiele}

Der zweite Ansatz stellt die informelle Förderung bestimmter Kompetenzen durch den Umgang mit Computerspielen in den Vordergrund der Betrachtung. Die theoretische Ausgangsüberlegung dabei ist, dass Computerspiele Aufgaben und Anforderungen beinhalten, deren Bewältigung Kompetenzen erfordern, die beim Spielen selbst erworben werden können und müssen. Da die Spielenden bestrebt sind, im Spiel zu bleiben bzw. es zu gewinnen, investieren sie nicht unerhebliche Zeit und Anstrengungen, um die Herausforderungen der digitalen Spiele verstehen und meistern zu können. Diesen Zusammenhang zwischen Computerspielen und Kompetenzerwerb hat Patricia M. Greenfield schon in ihrem 1984 erschienenen Buch «Mind and Media» thematisiert. Sie hat dort aufgezeigt, welche komplexen kognitiven Anforderungen mit scheinbar simplen Computerspielen wie Pac Man verbunden sind, und die These vertreten, dass solche Spiele induktive Lernprozesse anregen und bemerkenswerte kognitive Fähigkeiten schulen. Diese These hat Greenfield in späteren Publikationen auch mit empirischen Daten untermauert und insofern erweitert und spezifiziert, als sie von der Herausbildung von «inductive discovery skills» ausgeht (Greenfield et al. 1996). Das bedeutet, im Zusammenhang mit der beständigen Zunahme des Wissens über Regeln und Strukturen eines Spiels entsteht auch ein Wissen darüber, wie man sich diese Regeln und Strukturen erschliessen kann, und bei der Entwicklung von Lösungswegen und Strategien für die Bewältigung von Spielaufgaben entsteht zugleich ein Wissen über Aufgabentypen und darauf bezogene Erfolg versprechende Hand- 
lungsmuster und Strategien. Pädagogisch gewendet könnte man also sagen, dass hier gelernt wird, wie man entdeckend (d. h. induktiv) in einer virtuellen Umgebung lernt. Computerspiele tragen diesem Ansatz zu Folge also zur Herausbildung von umfassenderen und auf andere Kontexte übertragbaren kognitiven Kompetenzen bei, die zusammenfassend als «computer literacy» bezeichnet werden (Greenfield et al. 1996, S. 164). Diese unterscheide sich von traditionellen «literacy skills», wie sie für Printmedien benötigt werden, vor allem dadurch, dass sie eher visuell-räumliche als verbal-symbolische Fähigkeiten umfasse.

Diese Perspektive ist in den letzten Jahren häufiger eingenommen worden, prominent etwa in Jesper Juuls Entwurf einer Theorie der Computerspiele (Juul 2005), der das Spielen eines digitalen Spiels im Kern als eine Lernerfahrung bestimmt (vgl. auch Gebel/Gurt/Wagner 2004). Ihre Popularisierung dürfte mit verantwortlich sein für das aktuell zu verzeichnende Interesse an den sog. Serious Games und am Thema des Game Based Learning. Die genannten Autoren beschreiben durchaus komplexe und anspruchsvolle informelle Lernformen im Kontext des Spielens von Computerspielen. Gleichwohl tendieren auch sie dazu, das computerspielbezogene Lernen als Prozess zu charakterisieren, der eher auf einer unbewussten Ebene stattfindet und somit nicht jene reflexive Gestalt erhält, von der wir bei Bildungsprozessen ausgehen wollen.

Bei der Frage nach den Implikationen von Computerspielen für Lern-, Bildungsund Sozialisationsprozesse spielen - explizit oder implizit - ihre spezifischen medialen Merkmale eine zentrale Rolle. Aufgrund ihrer ausgeprägten Interaktivität und weiterer formaler Gestaltungsmittel (z. B. im Bereich der Dramaturgie, der Inszenierung und der audiovisuellen Darstellung) werden Computerspiele als hochgradig immersive und involvierende Medienwelten angesehen, die den Spielenden sozusagen strukturell davon abhalten, eine kritische Distanz zum Geschehen und zu den eigenen Erfahrungen während des Spiels zu entwickeln. Im Unterschied zu dieser verbreiteten Sichtweise möchten wir im Folgenden aufzeigen, dass Computerspiele auch durch formale Gestaltungsmittel und -prinzipien charakterisiert sind, die komplexe und reflexive Lernprozesse, wie wir sie als Bildungsprozesse bezeichnen, ermöglichen oder sogar herausfordern können. Wir möchten dazu zwei Argumentationslinien skizzieren.

\section{Computerspielen als potenzielle Steigerung der Reflexivität}

Folgt man dem semiotischen Ansatz James Paul Gees, dann lassen sich die verschiedenen Bereiche der Lebenswelt als «semiotische Domänen» bezeichnen (Gee 2003). In modernen bzw. spätmodernen Gesellschaften ist es erforderlich, sich neben der Alltagswelt eine Vielzahl weiterer, spezieller semiotischer Domänen mit ihren je besonderen Handlungs- und Kommunikationsmodi zu erschliessen. Sich eine neue semiotische Domäne, also einen neuen Weltbereich zu erschliessen, bedeutet, eine jeweils neue Literalität zu erwerben. Im Falle der Computer- 
spiele ist das nach Ansicht von Gee eine spezielle, multimodale Variante einer «visual literacy» (ebd. S. 13). Wenn man die Literalität einer neuen semiotischen Domäne erwirbt, dann bedeutet das nach Gee aber auch, die Welt und sich selbst in einer neuen Weise zu sehen, also eine Steigerung von Reflexivität. Vor allem in den «non-lifeworld domains», also den nicht alltäglichen Bereichen, werden Beziehungen zu kulturellen Gruppen und Deutungsmustern entwickelt, die die Grenzen der eigenen Kultur überschreiten (ebd., S. 39). Die Auseinandersetzung mit den Prinzipien und Modi einer anderen semiotischen Domäne bringt die Erfahrung mit sich, dass die bisherigen Lern- und Denkstrategien dort nicht funktionieren, was zu deren Erweiterung oder Transformation Anlass gibt. Im Computerspiel geht die Begegnung mit anderen Weltsichten einerseits weiter als in anderen semiotischen Domänen, weil man hier z. B. die Identität eines anderen übernehmen kann, etwa die eines Mafiosi oder eines Palästinensers. Das bietet einen Anlass, sich der eigenen kulturellen Modelle von richtig und falsch bewusst zu werden, vor allem wenn die jeweils präsentierten kulturellen Modelle nicht zu beherrschend bzw. durchdringend sind, sondern auch parodiert und in Frage gestellt werden. Andererseits bleibt dieser Identitätswechsel gerahmt als Spiel, also als reversibel, zeitlich begrenzt und enthoben der im sonstigen Alltagsleben geltenden Folgen, so dass es leichter fällt, sich darauf einzulassen.

\section{Formale Gestaltungsmittel zwischen Immersion und Distanz}

Unser zweites Argument bezieht sich auf die oben referierte Kritik, dass immersive und involvierende Computerspiele keine reflexive Distanz zuliessen. Unseres Erachtens zeigt ein genauerer Blick auf das Phänomen ganz im Gegenteil, dass es durchaus spielimmanente mediale Strukturen und Gestaltungsmittel gibt, die Reflexivität anregen, und zwar in dem Sinne, dass die Spieler einerseits Teil eines Spielgeschehens werden, zugleich jedoch auch eine reflexive Ebene behalten. Unsere Beobachtung ist also die, dass Thematisierungsformate und Gestaltungsmittel existieren bzw. entwickelt werden können, mit deren Hilfe immersive Tendenzen irritiert bzw. unterbrochen werden, durch die Mediennutzer dazu angeregt werden, eine reflexiv-kritische Distanz zum medial Präsentierten aufzubauen, und zwar ohne dass dies den Spielspass beeinträchtigt. Dies verstehen wir als eine Struktureigenschaft von Computerspielen. Ohne Anspruch auf Vollständigkeit sehen wir folgende verbreitete Spielelemente als distanzierende Formelemente von Spielen:

a) Lernhilfen für den Spielenden: Fast alle neueren, komplexen Spiele enthalten unterschiedliche Lernhilfen, die mehr oder weniger gut in die Spielwelt bzw. Spielgeschichte integriert sind. Matthias Bopp beispielsweise hat sowohl die "getarnten» als auch die offeneren Formen der in Computerspielen enthaltenen didaktischen Unterstützung der Spielenden am Beispiel des Spiels Silent Hill 2 (Konami 2003) aufgezeigt (Bopp 2004). Der Spieler findet z. B. in der Spielwelt eine Notiz mit nütz- 
lichen, später benötigten Informationen, oder er begegnet einem «Non-PlayerCharacter», der ihm hilfreiche Tipps für die nächste Mission gibt. Andere Spiele bieten komplexe Tutorials, die bisweilen in Modulen organisiert sind (beginnend mit einfachen Aufgaben, endend mit schwierigeren). Weil diese Lernhilfen mehr oder weniger plausibel in das Spielgeschehen integriert erscheinen, wird die «immersive Didaktik» der Computerspiele zumeist nicht als pädagogische Einwirkung wahrgenommen. Gleichwohl produzieren gerade Tutorials oder im Spiel vorhandene NPC-Ratgeber eine Spannung zwischen der Immersion in die Spielwelt und der Notwendigkeit, zugleich auf diese aus einer «informierten` Distanz zu schauen. Im Kontext der handlungstheoretischen Spieltheorie Leontjews (1980, S. 377ff.) kann man diese Spannung mit der Differenz von Handlung und Operation erklären, die als Strukturmerkmal von Spieltätigkeiten anzusehen ist. Mit Spielhandlung werden die gespielten Handlungen (z. B. Auto fahren, ein Raumschiff steuern) bezeichnet, mit Spieloperation dagegen die Tätigkeiten, die der Spielende tatsächlich ausübt (z. B. mit einem Spielzeugauto hantieren oder einen Joystick bedienen).

b) Ironie: Das ironische Spiel mit Bedeutungen ist eine weitere Strategie, die in vielen digitalen Spielen gefunden werden kann (zu Begriff und Bedeutung der Ironie in komplexen Gesellschaften vgl. Rorty 1989, S. 73). Als ein prominentes Beispiel sei Doom II genannt (id-Software 1994). In diesem Spiel kann man einen Raum entdecken, in dem drei gleich aussehende, kindlich erscheinende Figuren an der Decke baumeln (und abgeschossen werden können), in denen Eingeweihte den Hauptcharakter eines älteren Spiels wieder erkennen, nämlich Billy Blaze aus dem Spiel Commander Keen (id-Software 1991). Während der Unkundige das Geschehen lediglich oberflächlich (also den Präsentationsraum) betrachtet und das Abschiessen einer wehrlosen kindlichen Spielfigur als inakzeptabel beurteilen wird, werden erfahrene Spieler dieser Situation eine andere Bedeutung geben und sie als ironische, mit schwarzem Humor vermischte, Art des Abschiednehmens von einem Helden ihrer Kindheit (und den frühen Tagen des Computerspielens) dechiffrieren. Das visuelle Zitat dieses Spielcharakters verweist aus dem Spiel heraus auf einen anderen medialen Text - bzw. eine andere «semiotische Domäne» im Sinne Gees - und steht damit für die Pluralität der vorhandenen medialen Welten. Dieses Zitat zerstört zwar nicht das Spiel, provoziert aber durchaus eine kurze Irritation.

c) Spannung zwischen Regeln und Fiktion: Jesper Juul (2005) hat digitale Spiele als eine spezifische Kombination von Regelsystem und fiktiver Welt beschrieben. Der Bildschirm (re-) präsentiert mithin zwei unterschiedliche Arten von Informationen: Einerseits erscheint die (fiktionale) Spielwelt mit ihren Charakteren, Landschaften, Objekten etc. auf der Bildfläche. Andererseits wird der Spieler über seinen Status (bzw. den seiner Spielfigur) und seine Aufgaben, über mögliche und nicht mögliche Spielzüge sowie über verfügbare Ressourcen informiert. Anders formuliert: 
Ein grosser Teil des Interfaces ist auf dem Bildschirm selbst platziert, wo es von der zugleich dargestellten Spielwelt zu differenzieren ist. Während in den klassischen Adventure- und auch Ego-Shooter-Spielen auf dem Bildschirm noch häufig eine klare Aufteilung zwischen Spielwelt (obere zwei Drittel) und Spielsteuerung (unteres Drittel) zu finden war, überlagern die Interface-Elemente inzwischen zunehmend die Darstellung der Spielwelt. Diese grafischen Einblendungen stören auf gewisse Weise die Präsentation der Spielwelt - zumindest aber gehören sie zu einer anderen Informationsebene, auf die der Spieler ebenso Acht geben muss wie auf das Spielgeschehen selbst. Etablierte Beispiele dafür sind eingeblendete Displays, die über den Gesundheitsstatus der Spielfigur, die verbleibende Munition, verfügbare Waffen, Ausrüstungsgegenstände oder andere Ressourcen informieren oder auch Navigationshilfen (Karten, Kompasse). Man denke aber auch an Phänomene wie goldene Münzen oder Sterne, die in einer Spiellandschaft verstreut herum liegen und zur Verbesserung des Highscores eingesammelt werden können, und deren Bedeutung nicht aus der Spielwelt heraus, sondern nur im Kontext der Spielregeln erschlossen werden kann. Jesper Juul spricht mit Blick auf diese spezifischen Merkmale digitaler Spiele von der «Inkohärenz von Fiktion und Regeln». Die Regelzeichen erzeugen eine zweite Bedeutungsebene, die nicht nur für eine kurze Zeit präsent ist (wie etwa die oben erwähnte ironischen Zitate), sondern die zumeist die ganze Zeit über sichtbar bleiben. Der Spieler ist gehalten, kognitiv ständig zwischen der fiktionalen und der extrafiktionalen Spielebene, zwischen der Darstellung der Spielwelt und den Icons, Karten und Navigationstools des Interface umzuschalten, was einer bruchlosen Immersion in die fiktionale Spielwelt zuwiderläuft.

d) Irritation konventioneller Wahrnehmungs- und Aktionsweisen: Als letztes Beispiel spieldistanzierender Strukturelemente führen wir ein Element an, das ähnlich in vielen Science-Fiction-Filmen gefunden werden kann. Dieses Genre inszeniert häufig Welten, welche unsere konventionellen Wahrnehmungsweisen (so etwa die irdische Alltagsphysik) aushebeln und ihr somit eine imaginäre Alternative entgegensetzen. Dasselbe trifft für Computerspiele zu. Max Payne 2 beispielsweise (Remedy/Rock Star 2003) erlaubt dem Spieler einen so genannten «Bullet Time»-Modus zu aktivieren, in welchem alle Bewegungen in extremer Zeitlupe ablaufen. Da der Spieler weiterhin in Echtzeit agieren kann, erhält er dadurch einen erheblichen Vorteil gegenüber seinen virtuellen Gegnern. Dieses ungewöhnliche ästhetische Element ist aus dem Film The Matrix (Warner Bros. 1999) bereits bekannt, bricht aber doch mit unseren konventionellen Wahrnehmungsweisen. Sie sind geeignet, das Vertrauen in den authentischen Wahrheitsgehalt von Bildern zu begrenzen, indem sie ihren konstruktiven Charakter sicht- und erfahrbar machen. Mehr noch als dies, enthält eine solche Aufhebung und imaginäre Transgression der alltagsweltlichen Physik durchaus das Potenzial, Rahmenwechsel zu provozieren, so etwa eine Idee der Relativität von Zeit hervorzurufen. Im Bereich der digitalen Spiele 
kommt hinzu, dass solche Phänomene auch handlungsrelevant werden (können), wie bei Max Payne.

Die hier angeführten Beispiele zeigen, dass in digitalen Spielen Techniken und Elemente beobachtbar sind, die einen Zustand der «reflexiven Immersion» ermöglichen, in welchem der Spieler zugleich die spielimmanente wie auch die externe Perspektive einnimmt und auf diese Weise eine Distanz zur rein spielimmanenten Ebene erzeugt. Daraus wird ersichtlich, dass die Immersion in eine Spielwelt nicht per se mit dem Verlust des kritischen Bewusstseins einhergeht. Darüber hinaus enthalten die angeführten Strukturmerkmale vielfältige Anlässe zur Reflexion und zur Erweiterung der Selbst- und Weltsichten.

\section{Zur Bildungsrelevanz von Multiplayer-Spielen: Einblicke einer Fallstudie in die Counterstrike-Community}

Schon Singleplayer-Computerspiele weisen - wie gezeigt - Lern- und Bildungspotenziale auf. Für Multiplayer-Spiele gilt dies dort in verstärktem Masse, wo sich Spieler-Communities oder Netzwerke bilden. Eine im Jahr 2002/2003 durchgeführte empirische Untersuchung von Counterstrike-Spielern und ihren Aktivitäten brachte eine überraschende Bandbreite an Bildungs- und Lerneffekten ans Tageslicht. Am Beispiel des Multiplayer-Egoshooters Counterstrike (Valve 1999) wurde sichtbar, dass das Gamedesign einerseits und die Sozialität der Spieler-Community andererseits eng miteinander verwoben sind (Bausch/Jörissen 2004; Jörissen 2004; 2007). Die Einbindung des Einzelnen in die Community-Aktivitäten verläuft über Spieler-Clans, deren Mitglieder zwar in aller Regel durchaus «Fun»-orientiert sind, die jedoch zugleich die Angelegenheiten des Spiels und seiner Organisationsbedingungen (Turniere etc.) ausgesprochen ernst nehmen, und die in diesem Zuge auf einer Meta-Ebene des Spiels hohe soziale und moralische Standards etablieren (Fairness, angemessene Repräsentation des Clans, angemessene Konfliktlösungsstrategien etc.), welche das Spielgeschehen - d.h. den Spielhabitus der Einzelnen und der Gruppe - bestimmen.

Die Einführung von Counterstrike Ende der 1990er Jahre markiert den Anfang einer sehr speziellen Online-Spielerkultur. Zunächst einmal ist Counterstrike aus der Spieler-Community selbst hervorgegangen. Dieser extrem schnelle strategische Team-vs.-Team-Shooter ist eine Modifikation des damals berühmten SingleplayerSpiels Half Life (Valve 1998), bei der die Story, das Setting und die EinzelspielerOption entfernt wurden. Ein reines Multiplayer-Netzwerk-Spiel mit völlig neuen, von den Spielern selbst veränderbaren Landschaften und Spielmodi entstand auf diese Weise.

Da Counterstrike ausschliesslich für das Teamplay programmiert wurde und schwerlich ohne kollaborative Interaktion der Team-Mitglieder zu gewinnen ist, brachte dieses Spiel wie kaum eines zuvor Online-Spieler-Communities hervor, die sich regelmässig als Team (z. B. zu Trainingszwecken) treffen (online, aber nicht sel- 
ten auch offline). Die so genannten Clans sind die kleinsten sozialen Einheiten (mit i.d.R. fünf bis 50 Mitgliedern). Ein Austausch zwischen verschiedenen Clans erfolgt auf verschiedenen Webseiten (z. B. 4players.de), die einerseits der CommunityKoordination dienen, andererseits aber einen öffentlichen Kommunikationsraum bieten. Die Counterstrike-Community stellte sich als eine dichte, komplexe Verbindung von Spielern dar, die auf der sozialen Organisation und Interaktion auf Clan-Ebene basiert, sich von dort aus jedoch als weit verzweigtes soziales Netzwerk (über Clan-Verbindungen) ausbreitet.

\section{Die soziale Organisation der Counterstrike-Subkultur}

Clans sind zumeist demokratisch selbstorganisierte Einheiten, die kleinen Vereinen ähneln. In aller Regel geben sich Clans explizite Statuten. Dies bezieht sich etwa auf die soziographische Struktur der Clans (mit verschiedenen Rollen wie Clanleader, Schatzmeister, Team- oder Squadleadern, Vollmitgliedern, Juniormitgliedern und Mitgliedern auf Probe), aber auch auf Verhaltensregeln im Umgang innerhalb oder ausserhalb des Clans. Die Clanmitglieder leben üblicherweise in verschiedenen geographischen Regionen, so dass viele sich zuvor niemals offline getroffen haben. Sie treffen sich online, wenn sie zu festgelegten Zeiten am Training auf dem (eigens angemieteten) Clan-Server teilnehmen, wobei sie über ein spielinternes oder externes Voice-Chat-System miteinander kommunizieren. Strategien und taktische Spielzüge werden dann einstudiert und geübt, Spieler-Skills sowie interne organisatorische Angelegenheiten werden diskutiert. Nach dem Training (oder auch ausserhalb von Trainingsanlässen) treffen sich die Clanmitglieder häufig, um über Alltagsdinge zu sprechen. In der ethnographischen Rekonstruktion erwiesen sich die sozialen Beziehungen innerhalb des Clans häufig von Solidarität und emotionaler Bindung geprägt. Obwohl es unterschiedliche Grade der spielerischen Ambitioniertheit von Clans gibt - vom reinen «Fun»-Clan bis hin zum professionellen Clan - messen sich auch die weniger ambitionierten Clans regelmässig mit anderen Clans in so genannten "fun wars» oder im Rahmen offizieller Turniere. Durch diese Spielaktivitäten geraten Clans in engeren sozialen Austausch, woraus wiederum weitergehende Aktivitäten - wie etwa die gemeinsame Organisation und Veranstaltung einer LAN-Party - hervorgehen können. Die Online-Turniere ermöglichen auf diese Weise die Bildung sehr grosser und verzweigter Netzwerke auf Clan-Ebene, ohne welche der Zusammenhalt der Counterstrike-Community (als der vernetzte Verbund aus zehntausenden von Clans und Millionen von Spielern) nicht denkbar wäre. In unseren Gruppendiskussionen teilten uns die Spieler mit, dass sie mindestens hundert so genannter «Clan Tags» (also Kürzel von Clannamen) kennen oder zumindest wiedererkennen und dass sie sich an einzelne Online-Turniere noch nach Jahren erinnern würden. 


\section{Community-Werte und das Tabu des «Cheatens»}

In aller Regel achten Clans sehr darauf, dass ihre Mitglieder dem Ruf des Clans nicht schaden. Dies bezieht sich einerseits auf angemessene Umgangsformen und Verhaltensweisen sowohl innerhalb der Clanaktivitäten als auch im Kontakt mit anderen Clans; der Ruf eines Clans hängt aber vor allem davon ab, dass seine Mitglieder nicht «cheaten». "Cheatingtools» sind kleine Software-Programme, die es einem Spieler ermöglichen, im Spiel durch Wände zu sehen, niemals einen Gegner zu verfehlen, von Treffern weniger beeinträchtigt zu werden (geringerer Abzug von "Healthpoints») usw. - man kann dies in etwa mit Doping im Sport vergleichen, wobei die Schwelle zur Anwendung naturgemäss erheblich niedriger angesetzt ist. Es versteht sich, dass Spieler, die sich unlauterer Mittel bedienen, in keinem Spiel beliebt sind, unabhängig von der Art des Spiels. Doch im Fall der Counterstrike-Community ist dies ein hochgradig emotionalisiertes Thema, das in den Gruppendiskussionen sehr starke Reaktionen der Abwehr und Zurückweisung hervorgerufen hat. Cheating ist buchstäblich ein Tabu. Für die Konstitution der Counterstrike-Community spielt das Thema eine wichtige Rolle, insofern es einen Platz des Unberührbaren (sacer) definiert, und damit ein symbolisches Abgrenzungskriterium für in-group-Definitionen darstellt: Wer auch immer bei der Verwendung - oder auch nur beim Besitz - von Cheatingsoftware erwischt wird, muss damit rechnen, von den Spieleservern verbannt zu werden (die einzelnen Exemplare der Spiele tragen dazu eigens eine elektronische Signatur), aus seinem Clan ausgeschlossen zu werden und somit seine komplette Community-Identität und -Existenz zu verlieren. Was nun macht das Cheaten in diesem Kontext zu einem solchen Tabu? Cheaten entwertet die Anstrengungen und Bemühungen der anderen Spieler, ihr Spiel aus eigenem Engagement zu perfektionieren. Mehr noch: wer Cheatingsoftware benutzt, wird im Spiel allmächtig, "god-like». Dies aber widerspricht der Funktionsweise eines Spiels, das gerade darauf basiert, dass die einzelnen Spieler individuelle Freiheiten opfern, indem sie diese den Kollaborationsanforderungen des Teamplays übereignen. Diese Erfahrung von Emergenz, vom Aufgehen in einem im Idealfall wie ein «flow» funktionierendem Ganzen, machte für viele der beforschten Spieler/innen die Faszination dieses Spiels aus (die Geschwindigkeit von Counterstrike und die Reibungslosigkeit der Spielabläufe - wenn hinreichend einstudiert - dieses sehr schnellen Spiels spielt hierbei eine massgebliche Rolle). Das Cheaten ist ein direkter Angriff auf diese wichtige und die Mitglieder miteinander verbindende Erfahrungsebene - und somit auf die Community selbst. 


\section{Mit anderen Augen sehen: die subkulturelle Konstruktion der visuellen Bedeutungsgehalte des Spiels}

Insofern Computerspiele vor allem auf visuellen Gehalten basieren - wenn es sich auch um virtuelle, interaktive Formen von Bildern handelt - muss die Analyse von Computerspielen ihre visuelle Ebene berücksichtigen. Die oben erwähnten Ebenen - Oberfläche, Handlungsebene und Code-Ebene - überlagern sich zu der jeweils bestimmten semiotischen Struktur, die, wie wir feststellen konnten, vor allem eine soziale und subkulturell konstruierte ist. Während Nicht-Spieler regelmässig den visuellen Gehalt der Spiele ohne Berücksichtigung des sozialen Kontexts beurteilen - also ein naives Urteil fällen, wie es häufig in der oberflächlichen Kritik von Computerspielen geschieht -, konstituiert sich für die Spieler selbst die Bedeutung der Visualität des Spiels gerade nicht - zumindest nicht nur, und im Fall von Counterstrike sogar kaum - auf der «sichtbaren» Oberfläche, sondern vielmehr auf der Ebene der Interaktionen und der spezifischen, durch die Spieler-Community hervorgebrachten Bedeutungszuweisungen.

Die Visualität des Spiels ist aber nicht nur auf der Bedeutungsebene sozial determiniert. Im besonderen Fall des Computerspiels ist das Bild in jedem Augenblick unmittelbares Ergebnis der (Spiel-) Handlungen aller Beteiligten einerseits sowie der Algorithmen der Game- und Grafikengine andererseits (Bausch/Jörissen 2005). Beide Momente bilden eine unauflösbare sozio-technologische Verbindung. Insofern stellt aus unserer Perspektive die performative Dimension des Spiels, wie sie sich im Vollzug durch die Spielenden ereignet, ein essentielles Element der Interpretation der ikonologischen Gehalte des Spiels dar.

Im Fall von Counterstrike erweisen sich die Repräsentationen von Körpern, Tod und Raum als Schlüsselaspekte für das Verständnis seiner ikonologischen Gehalte. Auch wenn die Tötungshandlungen auf den ersten Blick das dominante Moment im Spiel zu sein scheinen, zeigt eine komparative Analyse des Körpermodells, insbesondere die Inszenierung des Sterbens (der getroffenen Avatare), dass die Körper in Counterstrike erstens eher einer Logik des Mechanischen denn des Fleischlichen folgen, und dass infolge dessen der inszenierte Tod in der Analyse geradezu gegenteilig als «aufgehobener Tod» sichtbar wird: Eigenschaften realer Sterblichkeit - wie Verletzlichkeit, körperliche Fehlfunktionen, die Unbestimmtheit und Transitivität des Sterbens als ein Zustand irgendwo zwischen Leben und Tod, sowie die Endgültigkeit des Todes - all diese Eigenschaften fehlen beinahe völlig in der interaktiven Bildlichkeit dieses Spiels - durchaus im Gegensatz zu anderen Ego-Shootern wie etwa dem berüchtigten, von der US-amerikanischen Armee vertriebenen «America's Army» (2002). Im Vergleich zu anderen Shooter-Spielen erscheint die Repräsentation von Tod und Sterben auffällig schwach - zugunsten einer Konzentration auf das "Gameplay», also den schnellen Spielablauf, in dem visuelle Details nur stören würden (sowohl auf der Wahrnehmungsebene als auch aufgrund der für den Bildaufbau benötigten Rechenzeit). Die Spiel-Körper zeigen 
keine Zeichen von Verletzung; sie sind bis zum letzten «Healthpoint» gleichermassen reaktions- und einsatzfähig. In ihrer geradezu maschinellen Körperästhetik verkörpern sie eher einen Typus des mechanischen, männlichen Helden, wie er in vielen Filmen auftritt, also eher eine übermenschliche als eine menschliche Figur. Verlieren die Kämpfer ihren Healthpoint, brechen sie unter kurzem Aufstöhnen «tot» zusammen. Bei all dem ist der virtuelle Tod der Spielfigur nichts, was vermieden werden müsste; vielmehr obliegt die Entscheidung darüber der angewandten Strategie und der jeweiligen strategischen Rolle des Spielers. Bewundernd berichteten die von uns beforschten Spieler von jenem Mitglied des «Pro-Clans» MouseSport, der in jeder Spielrunde als erster stirbt, weil er dadurch seinem Team die beste strategische Position verschafft - sein Verzicht auf Spielzeit und auf den unmittelbaren «Ruhm», im siegreichen Moment dabei zu sein, lässt ihn zu einem Mythos der Gemeinschaft werden, deren Ethos des Teamplays darin deutlich zum Ausdruck kommt.

Klarheit, Strategie und Kontrolle sind weitere Eigenschaften der virtuellen Umgebung des Spiels. Die virtuellen Areale - so genannte «Maps» - sind, trotz ihrer grossen, von Usern bereitgestellten Anzahl ästhetisch relativ austauschbar (abgesehen vielleicht von einer speziellen Variante von «Fun»-Maps, in denen es häufig darum geht, eine möglichst groteske Umgebung zu präsentieren, die sich allerdings zum ernsthaften Spiel nur bedingt eignet und daher in der Spielpraxis kaum verwendet wird). Bereits der Ausdruck «Maps» zeigt die reduzierte Wahrnehmung der diversen virtuellen Gelände an: es kommt auf die strategischen Optionen (Verstecke, Hindernisse etc.) an, die eine Map bietet. Der springende Punkt dieses Teamplay-Shooters liegt darin, dass die Teammitglieder die virtuellen Kampfarenen bis ins kleinste Detail kennen. Die Umgebung des Spiels wird, wie die Gruppendiskussionen zeigten, nicht so sehr als individuelle visuelle Erfahrung, sondern vielmehr als strategischer Raum, der eine reibungslose Koordination des Teams ermöglicht, wahrgenommen. Das Spiel findet, wie die Spieler es ausdrückten, «im Kopf» statt, auf einer geistigen Karte, die von allen Teammitgliedern internalisiert wurde.

$\mathrm{Ob}$ es sich um einen Ego-Shooter oder um eine andere Variante des digitalen Multiplayer-Spiels handelt: die Erfahrung, einen Bedeutungsraum gemeinsam geteilter Interpretationen der spielimmanenten Elemente wie auch der Regeln auf der Metaebene des Spiels gemeinschaftlich zu erschaffen, ist aus unserer Sicht ein sehr bildungsrelevantes Geschehen (im Sinne der Aneignung neuer Welt- und Selbstsichten). Dieser kollaborative Geist - verstanden als der Ort, an dem die Bedeutung des Spiels sozial konstruiert und an neue Mitglieder weitergegeben wird - erzeugt den Sinn des Spiels. Zugleich aber ist die «semiotische Domäne» des Spiels alles andere als nur sozial (durch eine jeweilige Gruppe von Spielern) determiniert. Denn diese Konstruktionen setzen auf den strukturellen Elementen des Spiels auf: der Benutzeroberfläche, den einprogrammierten Algorithmen, Regeln, 
Handlungsoptionen, der Navigation, dem visuellen Design etc., also dem, was Gameplay und Gamedesign ausmacht. Was pragmatistische Autoren wie George Herbert Mead schon vor hundert Jahren über die sozialisierende und vergemeinschaftende Bedeutung des gemeinschaftlichen Spielens feststellten (Mead 1934), scheint in unserer ludisch orientierten «digitalen Erlebniskultur» Kultur von wachsender Bedeutung zu sein (Simanowski 2008; Jörissen 2009).

\section{Schlussfolgerungen}

Unter der visuellen Oberfläche eines Computerspiels bilden seine Tiefenstrukturen - der Handlungsraum, der Coderaum sowie der das Spiel überschreitende soziale Raum - wichtige Bereiche, die bei der Beurteilung und Analyse von Computerspielen beachtet werden müssen. Bildungsaspekte lassen sich generell durch eine Analyse auf diesen Ebenen auffinden, also in den zwei Dimensionen a) der Interaktions- und Interfacestruktur sowie b) der sozialen Interaktionsstruktur im Umfeld des Spiels. In der ersten Dimension geht es um ein «close reading» der Ebenen

Tabelle 1 soll die hier diskutierten Zusammenhänge noch einmal in einer Übersicht verdeutlichen. Zeile (1) bezieht sich auf die Dimension der Selbstreferenz (Identität), Zeile (2) auf die Weltreferenz (Kulturalität und Sozialität), Zeile (3) auf Rahmungs- bzw. Umrahmungskompetenzen (z. B. Ironie) und Zeile (4) auf die Erfahrung von Differenz in Form anderer imaginativer und symbolischer Welten.

\begin{tabular}{|c|c|c|}
\hline $\begin{array}{l}\text { Dimensionen der Bildungs- } \\
\text { relevanz }\end{array}$ & $\begin{array}{l}\text { Interface- und Interaktions- } \\
\text { ebene (s. Abschnitt 3) }\end{array}$ & Soziale Ebene (s. Abschnitt 4) \\
\hline $\begin{array}{l}\text { 1. spielerische Aneignung und } \\
\text { Erweiterung von Identitäten }\end{array}$ & $\begin{array}{l}\text { Rollenübernahme von Spielcharak- } \\
\text { teren } \\
\text { Perspektive anderer Spieler überneh- } \\
\text { men } \\
\text { spielerisches «Als-ob»-Handeln }\end{array}$ & $\begin{array}{l}\text { «in-group» und «in-game»-Identi- } \\
\text { täten erhalten und aushandeln }\end{array}$ \\
\hline $\begin{array}{l}\text { 2. Aneignung und Erweiterung } \\
\text { der Weltsichten und -relati- } \\
\text { onen }\end{array}$ & $\begin{array}{l}\text { Wiedererkennen intermedialer Zitate } \\
\text { im Spiel (aus anderen Spielen, Filmen } \\
\text { oder Medien) } \\
\text { «in-game»-Tutorials und Informati- } \\
\text { onen } \\
\text { «immersive Didaktik» }\end{array}$ & $\begin{array}{l}\text { Teilnahme an neuen sozialen Um- } \\
\text { gebungen (Clans, Tribes einer be- } \\
\text { stimmten Spieler-Community) }\end{array}$ \\
\hline $\begin{array}{l}\text { 3. Aneignung von Rahmungs- } \\
\text { kompetenzen }\end{array}$ & $\begin{array}{l}\text { Ironisches Spiel mit Bedeutungen } \\
\text { visuelle Rahmung durch Onscreen- } \\
\text { Menüs und Icons }\end{array}$ & $\begin{array}{l}\text { Umrahmen visueller Bedeutungen } \\
\text { im Einklang mit den Normen der } \\
\text { Community } \\
\text { aktives Umarbeiten der Bildgehalte } \\
\text { des Spiels (häufig ironisch, z. B. Ma- } \\
\text { chinima) }\end{array}$ \\
\hline $\begin{array}{l}\text { 4. Dezentralisierung, Unter- } \\
\text { schiedliche Weltsichten er- } \\
\text { fahren }\end{array}$ & $\begin{array}{l}\text { Alternative Wahrnehmungsmodi (z. B. } \\
\text { «Bullet Time») }\end{array}$ & $\begin{array}{l}\text { Erfahrung alternativer Perspektiven, } \\
\text { Habitus, sozialer und/oder kulturel- } \\
\text { ler Differenzen zwischen Peers und } \\
\text { Community-Mitgliedern }\end{array}$ \\
\hline
\end{tabular}


der Software, der Interfaceelemente und der Interaktionsmodi. In der zweiten Dimension ist eine Analyse der involvierten Gaming-Subkulturen (etwa durch Gruppendiskussionen mit Spielern, Teilnehmende Beobachtung oder Diskursanalyse von Online-Netzwerken und Spieler-Foren) angezeigt.

\section{Weiterführende Implikationen}

Das Aufkommen digitaler Spiele und ihre Integration in unsere Lebenswelten bringen neue Anforderungen an die Individuen mit sich. Bezüglich der Neuen Medien und insbesondere virtueller Umgebungen mit ihren einzigartigen sozialen und kulturellen Strukturen sind Medienkompetenz und Medienbildung in verstärktem Masse gefordert. Dies schliesst die Fähigkeiten ein,

- in virtuellen Räumen zu navigieren,

- an den verschiedenen Formen von Online-Sozialität zu partizipieren,

- mediale Inhalte und Umgebungen zu entwickeln und zu designen, sowie

- in virtuellen Umgebungen gemachte Erfahrungen in die individuelle Erfahrungswelt sinnvoll zu integrieren.

Das Bildungssystem kann dazu beitragen, diesen gehobenen Anforderungen der Zukunft gerecht zu werden, indem es virtuelle Räume wie Computerspiele und soziale Online-Netzwerke in die «realen» pädagogischen Handlungsräume integriert. Dazu ist es aus unserer Perspektive notwendig,

- die pädagogische Diskussion über digitale Spiele inklusive der Spieler-Kulturen als bildungsrelevante Phänomene auf eine breitere Basis zu stellen,

- digitale Spiele als Umgebungen für informelle Lernprozesse und als mögliche Katalysatoren für Meta-Lernen und Bildungsprozesse zu analysieren, sowie nicht zuletzt

- das Computer-Spielen als eine performative Praxis zu verstehen, die hochkomplexe soziale und subkulturelle Strukturen, Diskurse und Machtbeziehungen aufweist.

Die gegenwärtigen Debatten über den pädagogischen Gebrauch elektronischer Spiele betont vor allem die Entwicklung von pädagogischen Spielen oder Serious Games. Damit fokussiert sie hauptsächlich darauf, wie digitale Spiele dazu beitragen können, curriculare Lerninhalte zu transportieren (oder zumindest vordefinierte pädagogische Ziele Schülern/-innen oder anderen Zielgruppen nahezubringen). Im Hinblick auf die Bildungspotenziale elektronischer Spiele, wie wir sie in diesem Beitrag vorgestellt haben, plädieren wir dafür, die Sichtweisen auf das Phänomen Computerspiel deutlich zu erweitern und die pädagogischen Chancen digitaler Spiele in Bezug auf informelles und non-formelles Lernen anzuerkennen. Die gegenwärtig verbreiteten pädagogischen Angebote in Form didaktisch designter Spiele sollten durch solche pädagogischen Herangehensweisen ergänzt werden, 
welche die genuinen Bildungschancen und -wege, die dem alltäglichen Spielen und den Spielerkulturen innewohnen, erkennen, verstärken und begleiten.

\section{Literaturverzeichnis}

Arnseth, Hans Christian (2006). Learning to Play or Playing to Learn. A Critical Account of the Models of Communication Informing Educational Research on Computer Gameplay. In Game Studies, 6 (1). Retrieved September 19, 2007, from http://gamestudies.org/0601/articles/arnseth

Bausch, Constanze; Jörissen, Benjamin (2004). Erspielte Rituale. Kampf und Gemeinschaftsbildung auf LAN-Partys. In Christoph Wulf (Hg.), Bildung im Ritual. Schule, Familie, Jugend, Medien. (S. 303-357). Wiesbaden: VS Verlag für Sozialwissenschaften.

Bausch, Constanze; Jörissen, Benjamin (2005). Das Spiel mit dem Bild. Zur Ikonologie von Action-Computerspielen. In Christoph Wulf \& Jörg Zirfas (Hg.), Ikonologie des Performativen. (S. 345-364). München: Fink.

Behn, Rolf (1984). Videospiele als Abbild unserer Wirklichkeit. In Karl Josef Kreuzer (Hg.), Handbuch der Spielpädagogik. Band 3: Das Spiel als Erfahrungsraum und Medium. (S. 683-695). Düsseldorf: Schwann.

Bopp, Matthias (2004). Didaktische Methoden in Silent Hill 2. Das Computerspiel als didaktisch arrangierte Lernumgebung. In Britta Neitzel, Matthias Bopp \& Rolf F. Nohr (Hg.), «See? I'm real.». Multidisziplinäre Zugänge zum Computerspiel am Beispiel von ¿Silent Hill〉. (S. 74-95). Münster: Lit.

Coffield, Frank (2000). The necessity of informal learning. Bristol: Policy.

Council on Science and Public Health (CSAPH) (2007). Emotional and Behavioral Effects of Video Games and Internet Overuse (A-07). Retrieved October 10, 2007, from: http://www.ama-assn.org/ama/pub/category/17694.html

Dewey, John (1925). Experience and Nature. Chicago: Open Court.

Entertainment Software Association (ESA) (2007). Facts and Research // Game Player Data. Retrieved September 16, 2007, from: http://www.theesa.com/ facts/gamer_data.php

Faure, Edgar (1972). Learning to Be: The World of Education Today and Tomorrow. Paris: UNESCO.

Foley, Griff (1999). Learning in social action: A contribution to understanding informal education. London: Zed.

Fritz, Jürgen; Fehr, Wolfgang (1997) (Hg.). Handbuch Medien: Computerspiele. Bonn: Bundeszentrale für politische Bildung.

Fromme, Johannes, Meder, Norbert; Vollmer, Nikolaus (2000). Computerspiele in der Kinderkultur. Opladen: Leske + Budrich.

Fromme, Johannes (2006). Socialisation in the Age of New Media. In MedienPädagogik, 11. Retrieved July 16, 2007, from http://www.medienpaed.com/05-1/ fromme05-1.pdf 
Fromme, Johannes (2007). Spiele in virtuellen Umgebungen. Überlegungen zur Beschreibung und Analyse eines neuen Mediums. In Dietmar Hartwig, Christian Swertz \& Monika Witsch (Hg.), Mit Spieler. Überlegungen zu nachmodernen Sprachspielen in der Pädagogik. Norbert Meder zum 60. Geburtstag. (S. 9-27). Würzburg: Königshausen \& Neumann.

Fromme, Johannes (2008). Virtuelle Welten und Cyberspace. In Friederike von Groß, Winfried Marotzki \& Uwe Sander (Hg.), Internet - Bildung - Gemeinschaft. (S. 169-201). Wiesbaden: VS Verlag.

Gamm, Gerhard (2000). Nicht Nichts. Studien zu einer Semantik des Unbestimmten. Frankfurt am Main: Suhrkamp.

Gebel, Christa, Gurt, Michael; Wagner, Ulrike (2004). Kompetenzbezogene Computerspielanalyse. In medien + erziehung (merz), 48. Jg., Nr. 3, Juni 2004, S. 18-23.

Gee, James Paul (2003). What video games have to teach us about learning and literacy. New York: Palgrave Macmillan.

Goffman, Erving (1961). Asylums. Essays on the social situation of mental patients and other inmates. New York: Doubleday Anchor.

Greenfield, Patricia Marks (1984). Mind and media: The effects of television, video games and computers. Cambridge, Mass.: Harvard Univ. Press.

Greenfield, Patricia Marks; Cocking, Rodney (1996) (Ed.). Interacting with video. Nordwood, NJ: Ablex Publishing Corporation.

Hall, Stuart (1980). Encoding/Decoding. In Stuart Hall (Ed.), Culture, Media, Language. Working Papers in Cultural Studies 1972-79. (pp. 128-138). London: Routledge.

Hall, Stuart (1992). «New Ethnicities〉. In James Donald \& Ali Rattansi (Ed.), 〈Race〉, Culture and Difference. (pp. 252-259). London: Sage.

Harrison, Lesley (2003). A Case for the Underestimated, Informal Side of Lifelong Learning. In Australian Journal of Adult Learning, 43 (1), 23-42.

Hegel, Georg Wilhelm Friedrich (1977). Phenomenology of Spirit. New York: Oxford University Press.

Humboldt, Wilhelm v. (1993). The Limits of State Action. Indianapolis: Liberty Fund.

Jäckel, Michael (2008). Medienwirkungen. Ein Studienbuch zur Einführung. Wiesbaden: VS Verlag.

Jenkins, Henry (2006). Convergence culture. Where old and new media collide. New York: New York Univ. Press.

Jones, Steven (Ed.) (1997). Virtual culture. Identity and communication in cybersociety. London: Sage.

Jörissen, Benjamin (2004). Virtual Reality on the Stage. Performing Community at a LAN-Party. In Patric Hernwall (Ed.), Envision. The New Media Age and Everyday Life. (pp. 23-40). Stockholm: Stockholms Universitet. 
Jörissen, Benjamin (2009, erscheinend). Zukunft Bildung in der digitalen Erlebnisgesellschaft. In: Siebenhaar, Klaus/Schremper, Ralf (Hg.): Spielend lernen - Aspekte des game-basierten social learning. Berlin.

Jörissen, Benjamin/Marotzki, Winfried (2008, im Druck). Strukturale Medienbildung - eine Einführung. Begriffe - Methoden - Analysen. Bad Heilbrunn: Klinkhardt/ UTB

Juul, Jesper (2005). Half-real. Video games between real rules and fictional worlds. Cambridge, Mass.: MIT Press.

Law, Ian (1996). Racism, Ethnicity and Social Policy. London: Prentice Hall.

Leontjew, Aleksej Nikolajewitsch (1980). Probleme der Entwicklung des Psychischen. Königsstein/Ts.: Athenäum. 3. Auflage.

Marotzki, Winfried (1990). Entwurf einer strukturalen Bildungstheorie. Weinheim: Deutscher Studien-Verl.

Marotzki, Winfried (1996). Neue Konturen Allgemeiner Pädagogik. In Michele Borrelli \& Jörg Ruhloff (Hg.), Deutsche Gegenwartspädagogik. Band II. (S. 67-84). Baltmannsweiler: Schneider Verl. Hohengehren.

McMillan, Sally J. (2002a). A four-part model of cyber-interactivity. In New Media \& Society, 4 (2), 271-291.

McMillan, Sally J. (2002b). Exploring Models of Interactivity from Multiple Research Traditions: Users, Documents, and Systems. In Leah Lievfrouw \& Sonia Livingstone (Ed.), The Handbook of New Media. Social Shaping and Consequences of ICTs. (pp. 163-182). London: Sage.

Mead, Georg Herbert (1934). Mind, self and society from the standpoint of the social behaviorist. Chicago: University of Chicago Press.

Mead, George Herbert (1938). The Philosophy of the Act. Chicago: Univ. of Chicago Press.

Overwien, Bernd (1999). Informal Learning and Popular Education. In Adult Education and Development, 52, 165-178.

Poster, Mark (2006). Information Please. Culture and Politics in the Age of Digital Machines. Durham: Duke Univ. Press.

Raessens, Joost; Goldstein, Jeffrey (2005) (Ed). Handbook of Computer Game Studies. Cambridge, Mass.: MIT Press.

Rösler, Carsten (2004). Medien-Wirkungen. Münster: Westfälisches Dampfboot.

Rogge, Jan-Uwe (1985). Über die Bedeutsamkeit des Video- und Computerspiels im Medienalltag Jugendlicher. In Dieter Spanhel (Hg.), Das Spiel der Jugendlichen. (S. 100-117). Ansbach: Ansbacher Verlagsgesellschaft.

Rorty, Richard (1989). Contingency, irony, and solidarity. Cambridge: Cambridge Univ. Press.

Schulmeister, Rolf (1997). Grundlagen hypermedialer Lernsysteme. München: Oldenbourg. 
Simanowski, Roberto (2008). Digitale Medien in der Erlebnisgesellschaft. Kultur Kunst - Utopien. Reinbek b. Hamburg: Rowohlt.

Subrahamanyam, Kaveri; Greenfield, Patricia Marks (1996). The Effect of Video Game Practice on Spatial Skills in Girls and Boys. In Patricia Marks Greenfield \& Rodney Cocking (Ed.), Interacting with video. (pp. 95-114). Norwood: Ablex Publ. Corp.

Turkle, Sherry (1997). Life on the screen. Identity in the age of the Internet. London: Phoenix.

Unger, Alexander (2007). Technological Transformation of Education. In Reinhard Heil, Andreas Kaminski, Markus Stippak, Alexander Unger \& Marc Ziegler (Hg.), Tensions - Technological and Aesthetic (Trans)Formations of Society. (S. 213224). Bielefeld: transcript.

Vorderer, Peter; Bryant, Jennings (2006) (Ed). Playing Video Games. Motives, Responses, and Consequences. Mahwah, NJ: Lawrence Erlbaum.

Winnicott, Donald Wood (1990). The maturational processes and the facilitating environment. Studies in the theory of emotional development. London: Karnac. 